\title{
A Model for Comparative Ratings in Studies of Within-Family Differences
}

\author{
Lindon J. Eaves, ${ }^{1,2}$ Michael C. Neale, ${ }^{1}$ and Joanne M. Meyer ${ }^{1}$ \\ Received 6 July 1990-Final 15 April 1991
}

Comparative ratings between pairs of siblings or other relatives are commonly used to refine measures of intrafamily variation. A simple model, based on signal detection theory, is proposed which shows how comparative ratings can be used to estimate within-pair variances of true scores, which can, in turn, be modeled with any of the conventional approaches to partitioning genetic and environmental variance within families.

KEY WORDS: twins; siblings; comparative judgments; signal detection; heritability; Sibling Inventory of Differential Experience (SIDE).

\section{INTRODUCTION}

A very important strategy for the study of behavioral differences within families is the use of comparative ratings in which members of a family are compared with each other with respect to one or more traits. In the case of children, the comparisons may be made by parents. In the case of adults, for example, siblings or twins, subjects may be asked to rate themselves in comparison with the target relative and indicate whether they display "more" or "less" of the attribute than their sibling.

This approach has achieved justifiable prominence in the light of the repeated demonstration that the principal environmental factors in-

This research is supported by Grant GM-30250 from the National Institutes of Health.

${ }^{1}$ Department of Human Genetics, Medical College of Virginia, Richmond, Virginia 23298.

2 To whom correspondence should be addressed at Department of Human Genetics, Medical College of Virginia, P.O. Box 3, MCV Station, Richmond, Virginia 232980033. 
fluencing behavioral development, especially in the domain of personality, arise within families, rather than between families (see Plomin and Daniels, 1987; Eaves, Eysenck and Martin, 1989). The comparative strategy has been exploited, for example, in developing the Sibling Inventory of Differential Experience (SIDE; Daniels and Plomin, 1985) to operationalize ideas of intrafamilial environmental differences.

For all its obvious appeal as a way of refining distinctions within families, we have currently no approach to analyzing such comparative data which allows us to integrate such measures with the more familiar "absolute" ratings in a way which permits us to estimate genetic and environmental components of variance and covariance. This note outlines the elements of a theory of comparative measures, such as the SIDE, for the simplest case and shows how, if the theory can be justified, ratings of within-family differences may be translated into more familiar measures of within-family variance for absolute scales. The model thus provides a way of integrating comparative ratings within the familiar analytical framework of quantitative genetics.

\section{DATA STRUCTURE}

Here we consider only the simplest case. Pairs of relatives, such as twins, either rate each other reciprocally or are rated by two others (e.g., both parents) for a particular attribute. We assume that each subject is forced to decide whether he/she or a sibling displays "more" or "less" of the given attribute.

The raters, R1 and R2, thus rate the first and second siblings, S1 and $\mathrm{S} 2$, by deciding with respect to the given attribute whether $\mathrm{S} 1>\mathrm{S} 2$ or S1 < S2. It is essential for the application of the theory that two ratings be obtained for each pair. The basic structure of the $\mathrm{N}$ pairs of ratings is summarized in Table $I$.

The modeling task is to account for the pattern of pairwise comparative ratings in terms of (1) the process involved in making comparative judgments and (2) the magnitude of the "true" variation within

Table I. Basic Structure of Comparative Ratings of Relatives

\begin{tabular}{ccc}
\hline \multirow{2}{*}{ Rater } & \multicolumn{3}{c}{ Rater R1 } \\
\cline { 2 - 3 } R2 & $\mathrm{S} 1<\mathrm{S} 2$ & $\mathrm{~S} 1>\mathrm{S} 2$ \\
\hline S1 $<$ S2 & $N_{11}$ & $N_{12}$ \\
S1 $>$ S2 & $N_{21}$ & $N_{22}$ \\
\hline
\end{tabular}


pairs of subjects, so that we may summarize the variation within pairs in terms of familiar genetic and environmental components of variance.

\section{MODEL}

Figure 1 illustrates a theory, borrowed from Signal Detection Theory (SDT; see, e.g., Coombs et al., 1970), to account for how a typical first sibling, S1, arrives at a comparative rating of himself in relation to a second sibling, S2. The value of S1 on the latent attribute on which judgement is based is arbitrarily scaled to zero, and the trait value of the second sibling exceeds that of the first by D units. If $S 1$ is really greater than S2, D will be negative. The model assumes that the probability that $S 1$ will rate himself "less than" $S 2$ on the attribute increases monotonically with increasing difference between the trait values of the siblings, $D$. When $D=0$, the rater will say $\mathrm{S} 1<\mathrm{S} 2$ with probability 0.5 . As $D$ $\rightarrow \infty$, there will never be any doubt that $\mathrm{S} 1<\mathrm{S} 2$. Specifically, we assume that the probability of judgement $\mathrm{S} 1<\mathrm{S} 2$ follows the standardized cumulative normal probability integral thus:

$$
\Phi(D)=\int_{-D}^{\infty} \frac{1}{\sqrt{2 \pi}} e^{-1 / 2 x^{2}} d x .
$$

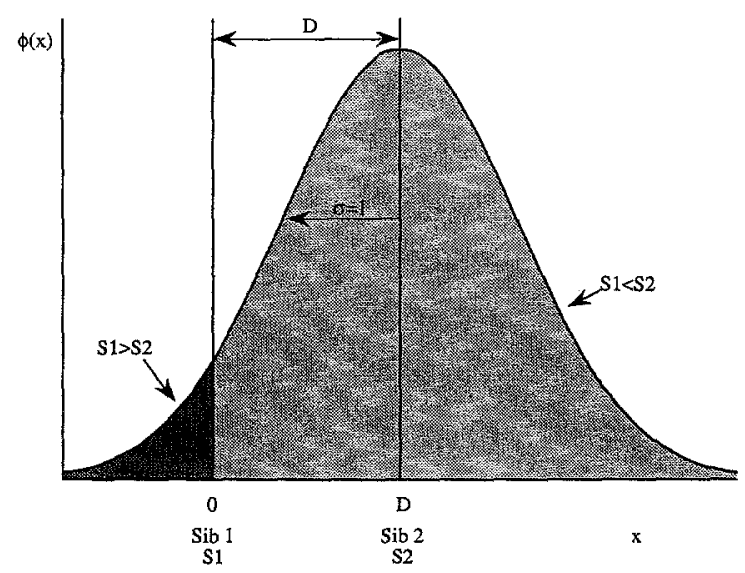

Fig. 1. Elements of a model for comparative judgments within sibling pairs. The normal curve represents the distribution of perceptions of S2's trait value by $\mathrm{S} 1$ around the true value of S2's trait relative to S1. 
If $\mathrm{S} 1$ is truly greater than $\mathrm{S} 2$, then $D$ will be negative on the current scale. For a given difference, $D$, between the trait values of $S 1$ and $S 2$, the four possible combinations of paired comparisons will have the probabilities given in Table II.

\section{THE LIKELIHOOD OF THE OBSERVED COMPARATIVE RATINGS}

In practice, we do not know the individual differences within pairs, $D$. However, if we can specify their distribution, we may nevertheless write the likelihood of the observed frequencies $N_{11} \ldots N_{22}$ and try to obtain maximum-likelihood (ML) estimates of the parameters of the "hidden" distribution of within-pair differences on which the comparisons are based. In this case, we make the usual assumption employed in the analysis of variance of differences within pairs of relatives that the intrapair differences are $N\left[0, \sigma_{\mathrm{w}}^{2}\right]$, where $\sigma_{\mathrm{w}}^{2}$ is the within-pair variance for the latent attributes. If we write

$$
\phi(D)=\frac{1}{\sigma_{w} \sqrt{2 \pi}} e^{\left(-\frac{1}{2} \frac{D^{2}}{\sigma_{w}^{2}}\right)},
$$

then the likelihoods of the four possible pairwise comparisons, assuming only that the intrapair differences are sampled from a normal distribution of such differences, may be derived as the weighted integral of the probabilities in Table II over all possible values of $D$. These likelihoods are given in Table III.

If we write $P_{11} \ldots P_{22}$ for the four individual likelihoods, then the log-likelihood of the overall set of $N$ pairwise comparisons is

$$
\begin{aligned}
L=\text { constant }+N_{11} \ln P_{11} & +N_{12} \ln P_{12} \\
& +N_{21} \ln P_{21}+N_{22} \ln P_{22},
\end{aligned}
$$

which may be evaluated for any $\sigma_{\mathrm{w}}^{2}$ and maximized with respect to that single unknown parameter. Although the expected frequencies in Table

Table II. Probabilities of Pairwise Comparative Ratings Assuming a Known Latent Difference Within Pairs, $D$

\begin{tabular}{ccc}
\hline & \multicolumn{3}{c}{ Rater R1 } \\
\cline { 2 - 3 } $\mathrm{R} 2$ & $\mathrm{~S} 1<\mathrm{S} 2$ & $\mathrm{~S} 1>\mathrm{S} 2$ \\
\hline $\mathrm{S} 1<\mathrm{S} 2$ & {$[\Phi(D)]^{2}$} & {$[\Phi(D)][1-\Phi(D)]$} \\
$\mathrm{S} 1>\mathrm{S} 2$ & {$[\Phi(D)][1-\Phi(D)]$} & {$[1-\Phi(D)]^{2}$} \\
\hline
\end{tabular}


Table III. Probabilities of Pairwise Comparative Ratings Assuming that Latent Differences Are Normally Distributed Values

\begin{tabular}{ccc}
\hline $\begin{array}{c}\text { Rater } \\
\text { R2 }\end{array}$ & \multicolumn{2}{c}{ Rater R1 } \\
\hline S1 $<$ S2 & $\int_{-\infty}^{\infty} \phi(D)[\Phi(D)]^{2} d D$ & $\mathrm{~S} 1>\mathrm{S} 2$ \\
$\mathrm{~S} 1>\mathrm{S} 2$ & $\int_{-\infty}^{\infty} \phi(D)[\Phi(D)][1-\Phi(D)] d D$ \\
& $\int_{-\infty}^{\infty} \phi(D)[\Phi(D)][1-\Phi(D)] d D$ & $\int_{-\infty}^{\infty} \phi(D)[1-\Phi(D)]^{2} d D$ \\
\hline
\end{tabular}

III look cumbersome, we note that highly accurate numerical approximations exist for the inner integral, $\Phi(D)$, and that the outer integral over the range $-\infty<D<\infty$ should pose little problem to standard programs for Gauss-Hermite quadrature (Numerical Algorithms Group, 1987).

\section{DISCUSSION}

A drawback of comparative ratings of a single type of relatives such as sibling pairs is our lack of a test of whether the perceived differences reflect any real underlying trait differences and whether such differences reflect responses to genetic or nongenetic factors. The first problem is solved by arranging for more than one comparative rating. The second is approached by the joint analysis of ratings on different kinds of relatives, e.g., $\mathrm{MZ}$ and $\mathrm{DZ}$ twins, reared together and apart. If the parameter $\sigma_{\mathrm{w}}^{2}$ is significantly greater than zero, we have evidence that the comparisons reflect genuine differences in latent trait values within families. The ML approach outlined above extends readily to provide tests of hypotheses about the relative values of $\sigma_{\mathrm{w}}{ }^{2}$ for different degrees of genetic and environmental relatedness and even to estimation of components of genetic and environmental variance within families (cf. Jinks and Fulker, 1970). Although the model is developed in terms of a naively simple comparative paradigm, we believe that the basic approach can be extended to more complex rating systems and, more importantly, to the inclusion of covariates of the comparisons which are measured on either comparative or absolute scales. Although the analysis is necessarily restricted to genetic and environmental variances within families, our approach does allow us to begin to specify how findings for comparative 
ratings can be integrated with those based on more conventional absolute measures familiar to statistical and behavioral geneticists.

\section{REFERENCES}

Coombs, C. H., Davies, R. M., and Tversky, A. (1970). Mathematical Psychology: An Elementary Introduction, Prentice Hall, Englewood Cliffs, NJ, Chap. 6.

Daniels, P., and Plomin, R. (1985). Differential experience of siblings reared in the same family. Dev. Psychol. 21:747-760.

Eaves, L. J., Eysenck, H. J., and Martin, N. G. (1989). Genes, Culture and Personality: An Empirical Approach, Academic Press, New York.

Jinks, J. L., and Fulker, D. W. (1970). Comparison of the biometrical, genetical, MAVA and classical approaches to the analysis of human behavior. Psychol. Bull. 73:311349.

Numerical Algorithms Group (1988). FORTRAN Library, Mark 12, NAG, Oxford.

Plomin, R., and Daniels, D. (1987). Why are children in the same family so different from each other? Behav. Brain Sci. 10:44-54.

Edited by D.W. Fulker 